\title{
Gravitational Wave Detection with Atom Interferometry
}

\author{
Savas Dimopoulos, ${ }^{1}$ Peter W. Graham, ${ }^{2,1}$ Jason M. Hogan, ${ }^{1}$ Mark A. Kasevich, ${ }^{1}$ and Surjeet Rajendran ${ }^{2,1}$ \\ ${ }^{1}$ Department of Physics, Stanford University, Stanford, California 94305 \\ ${ }^{2}$ SLAC, Stanford University, Menlo Park, California 94025
}

(Dated: December 8, 2007)

\begin{abstract}
We propose two distinct atom interferometer gravitational wave detectors, one terrestrial and another satellite-based, utilizing the core technology of the Stanford $10 \mathrm{~m}$ atom interferometer presently under construction. The terrestrial experiment can operate with strain sensitivity $\sim \frac{10^{-19}}{\sqrt{\mathrm{Hz}}}$ in the 1 $\mathrm{Hz}-10 \mathrm{~Hz}$ band, inaccessible to LIGO, and can detect gravitational waves from solar mass binaries out to megaparsec distances. The satellite experiment probes the same frequency spectrum as LISA with better strain sensitivity $\sim \frac{10^{-20}}{\sqrt{\mathrm{Hz}}}$. Each configuration compares two widely separated atom interferometers run using common lasers. The effect of the gravitational waves on the propagating laser field produces the main effect in this configuration and enables a large enhancement in the gravitational wave signal while significantly suppressing many backgrounds. The use of ballistic atoms (instead of mirrors) as inertial test masses improves systematics coming from vibrations and acceleration noise, and reduces spacecraft control requirements.
\end{abstract}

PACS numbers: 04.80.-y, 04.80.Cc, 03.75.Dg

The discovery of gravitational waves will open a new window into the Universe. Astrophysical objects such as black holes, neutron stars and white dwarf binaries which are difficult to observe electromagnetically are bright sources of gravitational radiation. Gravitational waves are unaffected by recombination and can therefore probe the earliest epochs of the Universe. Light interferometers have been at the forefront of gravitational wave astronomy. Recent advances in the control and manipulation of atoms have resulted in the development of a new generation of highly sensitive atom interferometers.

In this Letter, we propose terrestrial and satellite atom interferometer configurations to detect gravitational waves in the $10^{-3} \mathrm{~Hz}-10 \mathrm{~Hz}$ band. These are based on light pulse atom interferometry [1] in which a dilute ensemble of cold atoms in free fall is made to acrue a phase shift by the application of beamsplitter and mirror laser pulses along the direction of motion of the atoms. In this configuration, the atom interferometer serves as a Mach-Zehnder interferometer. The atomic transitions are triggered by pulses from a control laser at $x=0$ (dark gray in Figure 1) which are emitted at definite intervals of time $T$. When the pulse from the control laser hits the atom, the atom is already in the laser field emitted by a passive laser at $x=L$ (light gray in Figure 11). The atom then undergoes a 2-photon transition (e.g. via Raman scattering) with momentum transfer $k_{\text {eff }} \approx 2 k$ between the atom and the laser field where $k$ is the frequency of the laser. This changes the space-time paths of the atomic states. The phase shift in the atom interferometer arises from differences in the space-time paths of the interfering atomic states and the laser phase imprinted on the atom during the atom-laser interaction [2].

Signal.-A gravitational wave of amplitude $h$ and frequency $\omega$ modulates the laser ranging distance $L$ between two spatially separated points that lie on a plane perpendicular to the direction of propagation of the wave. The laser ranging distance oscillates with frequency $\omega$ and, when $\omega L \ll 1$, amplitude $h L$. In our setup described in Figure1, we separate the control and passive laser by $L$ and place two atom interferometers between them, one $\left(I_{1}\right)$ at $x_{1}$ near the control laser and the other $\left(I_{2}\right)$ at $x_{2}$ near the passive laser. Both interferometers are run using common lasers, enabling differential measurement strategies that drastically suppress systematics associated with the lasers [3].

The main signal of the gravitational wave in the interferometer at $x_{i}$ comes from laser phase from the passive laser, hence from the timing of these laser pulses. The control laser's pulses are always at $0, T$, and $2 T$. As an example, the first beamsplitter pulse from the control laser would reach the atom at time $x_{i}$ (setting $c=\hbar=1$ ) in flat space and so the passive laser pulse then originates at time $2 x_{i}-L$. However, if the gravitational wave is causing an expansion of space, the control pulse is 'delayed' and actually reaches the atom at time $\sim x_{i}(1+h)$. Then the passive laser pulse originated at $\sim\left(2 x_{i}-L\right)(1+h)$. Thus the laser phase from the passive laser pulse has been changed by the gravitational wave by an amount $k h\left(2 x_{i}-L\right)$. The differential phase shift between the interferometers is therefore $\sim k_{\text {eff }} h L$. A gauge invariant calculation of the differential phase shift due to a gravitational wave in the interferometer 


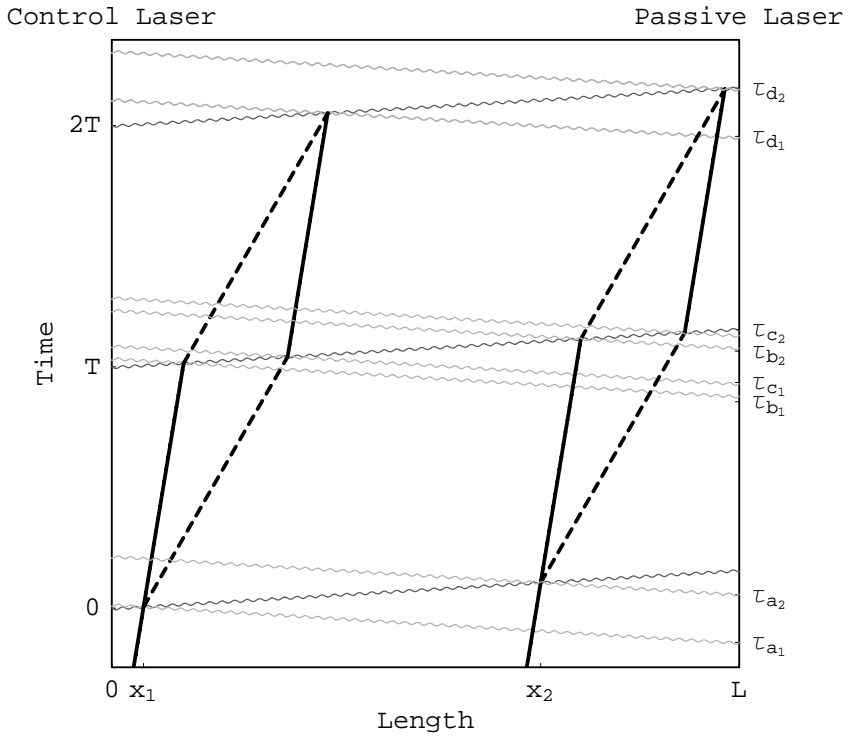

FIG. 1: A space-time diagram of two light pulse atom interferometers in the proposed differential configuration. The black lines indicate the motion of a single atom. Light from the control and passive lasers are incident from the left (dark gray) and the right (light gray) respectively.

configurations discussed above was performed in [4] and this calculation confirms the intuitive picture sketched in the above discussion. When $\omega L \ll 1$, the differential phase shift between the interferometers is given by:

$$
\Delta \phi_{\mathrm{tot}}=2 k_{\mathrm{eff}} h L \sin ^{2}\left(\frac{\omega T}{2}\right) \sin \left(\phi_{0}\right)
$$

where $\phi_{0}$ is the phase of the gravitational wave at the start of the interferometer which changes with time and leads to a time dependent phase shift in the interferometer.

This experiment can be viewed as a comparison of two spatially separated clocks in the presence of a gravitational wave. The two atom interferometers measure time at their respective location through the evolution of their phase. The comparison between the two clocks is performed using the laser pulses that execute the interferometry. In the presence of a gravitational wave, the propagation of these pulses is altered and produces a differential phase shift between the interferometers. The choice of the Mach-Zehnder control sequence to operate the interferometer is motivated by the need to eliminate Doppler effects present in atomic clocks.
Experimental Configurations.-Vibration noise severely limits the sensitivity of current gravitational wave detectors at frequencies below $40 \mathrm{~Hz}$. In the atom interferometer, the atoms are in free fall during the course of the interferometry and are coupled to ambient vibrations only through gravity. In addition to causing time variations in the local gravitational field, vibrations will also alter the launch position and velocity of the atoms. However, since the atom is in free fall when it is hit by the first beamsplitter pulse that causes the atom to accrue a phase shift, these vibrations do not directly lead to phase shifts except through their coupling with local gravitational fields. These effects are gravitationally suppressed and enable atom interferometers to probe low frequencies [4].

The lasers used to execute the interferometry are not inertial and their vibrations will alter their distance from the atoms, changing the emission times of the laser pulses. Since the phase of the laser pulse is imprinted on the atom during the atom-laser interaction, these vibrations will directly cause phase shifts in the interferometer. However, since both interferometers are run using common lasers, vibrational noise in the differential phase shift between the two atom interferometers is greatly suppressed. The pulses from the control laser at times 0 , $T$ and $2 T$ (Figure 1) are common to both interferometers and contributions from the vibrations of this laser to the differential phase shift are completely cancelled. The vibrations of the passive laser are also common except during the time $L$. The pulses from the passive laser that hit one atom interferometer $\left(\tau_{a_{1}}, \tau_{b_{1}}, \tau_{c_{1}}, \tau_{d_{1}}\right)$ are displaced in time by $L$ from the pulses $\left(\tau_{a_{2}}, \tau_{b_{2}}, \tau_{c_{2}}, \tau_{d_{2}}\right)$ that hit the other interferometer due to the spatial separation $L$ between the interferometers (Figure 1). However, unlike light interferometers, only the vibrations above frequencies $\frac{c}{L} \sim 3 \times 10^{4} \mathrm{~Hz}\left(\frac{10 \mathrm{~km}}{L}\right)$ are a background to the atom interferometer. These frequencies are higher than the frequency of the gravitational wave signal and are easier to suppress. Similarly, the differential measurement strategy ameliorates the control required over laser phase noise [4]. This measurement strategy significantly diminishes noise without impacting the signal.

Motivated by these considerations, we propose terrestrial and space based atom interferometer configurations that can search for gravitational waves in the $10^{-3} \mathrm{~Hz}-10 \mathrm{~Hz}$ band. On the Earth, one possible experimental configuration is to have a long, vertical shaft with the necessary apparatus to run one atom interferometer near the bottom and one near the top, as shown in Figure 2. The atom interferometers would be run vertically along the same axis as defined by the com- 


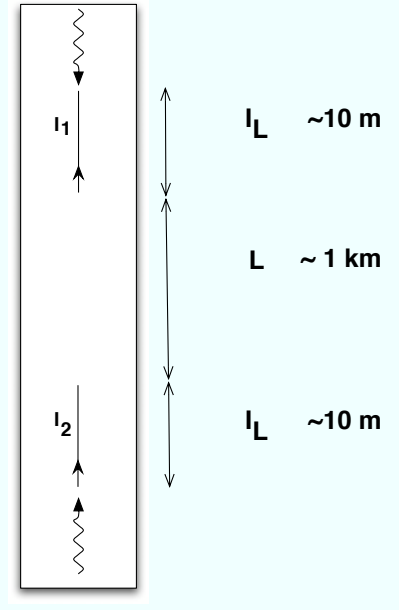

FIG. 2: A diagram of the proposed setup for a terrestrial experiment. The straight lines represent the path of the atoms in the two $I_{L} \sim 10 \mathrm{~m}$ interferometers $I_{1}$ and $I_{2}$ separated vertically by $L \sim 1 \mathrm{~km}$. The wavy lines represent the lasers.

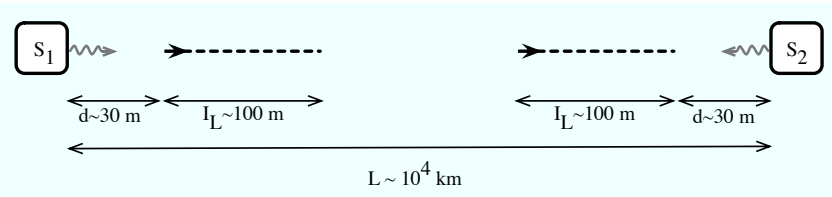

FIG. 3: The proposed setup for a satellite experiment. The dashed lines represent the paths of the atoms during the interferometer sequence. The atoms are launched from the satellites $S_{1}$ and $S_{2}$. The gray lines represent the lasers.

mon laser pulses applied from the bottom and top of the shaft. The two interferometers could be separated by baselines $L \sim 1-10 \mathrm{~km}$. If each atom interferometer is $I_{L} \sim 10 \mathrm{~m}$ long, the interferometer can allow for interrogation times $\sim 1$ second. In this configuration, the atom interferometer is sensitive to gravitational waves in the frequency band $1 \mathrm{~Hz}-10 \mathrm{~Hz}$.

Interrogation times larger than $1 \mathrm{~s}$ are difficult to achieve in a terrestrial, ballistic atom interferometer. The search for gravitational waves in the sub-Hertz band on the Earth is also impeded by time varying local gravitational fields. We are thus lead to consider satellite atom interferometer configurations to search for gravitational waves in the sub-Hertz band. Our configuration consists of two satellites in orbit separated by $L \sim 10^{3}-10^{4} \mathrm{~km}$. The satellites will act as base stations and run the atom interferometers along their axis using common laser pulses (Figure 3).
The atom interferometer requires the laser and the atom source to be placed in the satellite, near their power sources. However, the diffuse atom cloud trajectories that form the arms of the atom interferometer need not be located within the satellite. The space environment is predominantly composed of hydrogen at $\sim 10^{-11}$ Torr pressures with an ambient magnetic field $\sim 5 \mathrm{nT}$ that is correlated over $\sim 0.01 \mathrm{AU}[5,6]$. With a stabilizing magnetic field $\gtrsim 20 \mathrm{nT}$ provided by a permanent magnet housed in the spacecraft, this environment permits the operation of the atom interferometer out to distances $I_{L} \sim 100 \mathrm{~m}$ from the spacecraft and for interrogation times $\sim 2000 \mathrm{~s}$, limited by collisions of the atoms with interplanetary gas [4]. Prior to launch, the atoms must be positioned at distances $d$ and $d+I_{L}$ from their base stations $S_{1}$ and $S_{2}$ respectively using laser manipulations (Figure 3). The phase shift in the interferometer can be read using absorption detection by imaging the atom clouds with lasers. The $I_{L} \sim 100 \mathrm{~m}$ interferometer region will allow interrogation times $\sim 100 \mathrm{~s}$ for an atom interferometer operated with lasers delivering momentum kicks $k_{\mathrm{eff}} \sim 10^{9} \mathrm{~m}^{-1}$ using multi-photon atom optics [7, 8, 9, 10]. This detector is sensitive to gravitational waves with frequencies $\gtrsim 10^{-2} \mathrm{~Hz}$.

The gravitational tidal force on the test masses due to uncontrolled motion of the spacecraft is a major background for space-based gravitational wave detectors. Light interferometers like LISA require their test masses to be protected inside the spacecraft. In our proposal, the atoms are at a distance $d \sim 30 \mathrm{~m}$ from the spacecraft, reducing these tidal accelerations by $\sim 10^{4}[4]$ and relaxing the required position control of the satellite to $\sim 10 \frac{\mu \mathrm{m}}{\sqrt{\mathrm{Hz}}}$ for our predicted sensitivities, compared with LISA's $1 \frac{\mathrm{nm}}{\sqrt{\mathrm{Hz}}}$ requirement [11]. Furthermore, spurious electromagnetic forces due to charge transfer between the test masses and the satellite environment are a major background for LISA. Since the atoms are neutral and the atom interferometer is operated using magnetically insensitive $(m=0)$ atomic states, electromagnetic forces on the atom clouds are naturally small. Collisions of the atoms with the cosmic ray background lead to particle deletion from the cloud and not charging of the cloud. These deletions result in a minor reduction in the sensitivity (for interrogation times $\lesssim 1000 \mathrm{~s}$ ) but do not cause phase shifts.

Sensitivities.-The overall sensitivity of the atom interferometer is a function of the signal-to-noise ratio (SNR) of the interference fringes, the effective momentum transfer of the atom optics $\left(k_{\text {eff }}\right)$ and the distance between the interferometers. The SNR can be improved either with a larger number of atoms per cloud or by 


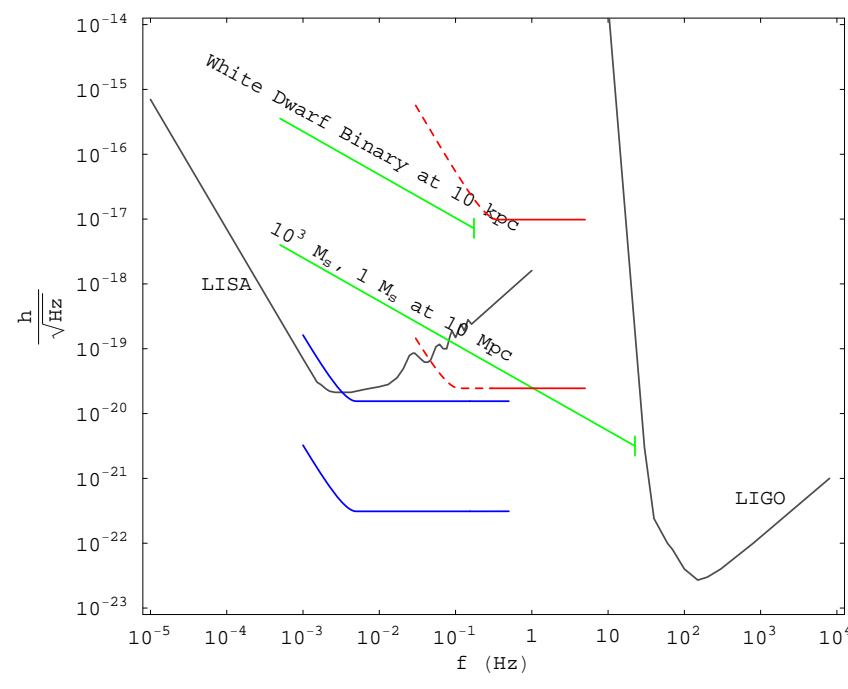

FIG. 4: The projected shot noise power spectra of our proposed terrestrial (red) and satellite experiments (blue) to a gravitational wave of frequency $f$. The two terrestrial setups use $k_{\text {eff }} \sim 10^{9} \mathrm{~m}^{-1}$ and $\sim 10^{10} \mathrm{~m}^{-1}$ beamsplitters, atom statistics noise $10^{-4} \mathrm{rad}$ and $10^{-5} \mathrm{rad}$ per shot with $1 \mathrm{~km}$ and $4 \mathrm{~km}$ baselines respectively. The satellite configurations describe setups with $k_{\text {eff }} \sim 3 \times 10^{9} \mathrm{~m}^{-1}$ and $\sim 10^{9} \mathrm{~m}^{-1}$ beamsplitters, $10^{3} \mathrm{~km}$ and $10^{4} \mathrm{~km}$ baselines and atom statistics noise $10^{-4} \mathrm{rad}$ and $10^{-5}$ rad per shot respectively. The sensitivity of the terrestrial setup is cut off (dashed) where it is below time varying gravity gradients. The terrestrial and space configurations assume a $10 \mathrm{~Hz}$ and $1 \mathrm{~Hz}$ data-taking rate respectively. Example sources are shown in green, enhanced by their lifetimes, ending when the binaries coalesce. $\mathrm{M}_{\mathrm{s}}$ refers to 1 solar mass.

using squeezed atom states [12, 13]. An atom statistics limited phase sensitivity (shot noise) of $10^{-4} \mathrm{rad}$ can be achieved, e.g. with cloud sizes around $10^{8}$ atoms, and $10^{-5}$ rad may be achievable in the near future. For the satellite based experiment, the baseline length is limited by the intensity of the lasers, the time available for the atomic beamsplitter and mirror transitions, and the rate of absorption and subsequent spontaneous emission driven by the intense beam from the near laser. With Rabi frequencies $\sim 10^{2}-10^{3} \mathrm{~Hz}$ for stimulated Raman transitions and a $1 \mathrm{~W}$ laser with a $1 \mathrm{~m}$ waist, $200 \hbar k$ beamsplitters $\left(k_{\text {eff }} \sim 10^{9} \mathrm{~m}^{-1}\right)$ should be achievable with up to $10^{4} \mathrm{~km}$ between the interferometers. The resultant sensitivities to gravitational waves are shown in Figures 4 and 5 .

The detection of gravitational waves at these sensitivities requires all time varying backgrounds at the frequencies of interest to be smaller than shot noise. Differ-

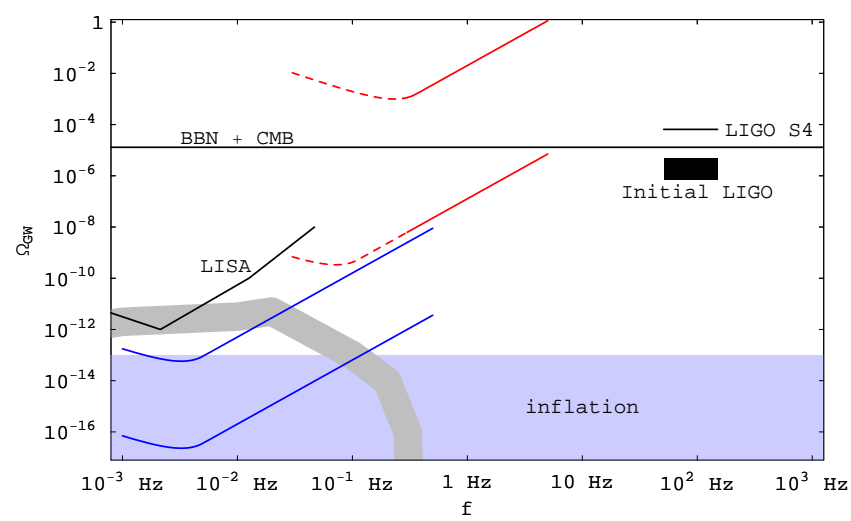

FIG. 5: The projected sensitivity in $\Omega_{\mathrm{GW}}$ of our proposed configurations from Figure 4 to a stochastic background of gravitational waves. These limits assume a year of integration using uncorrelated detectors. The gray band represents a prediction for the stochastic gravitational wave background from extragalactic White Dwarf binaries. The blue band shows the limit on gravitational waves produced in inflation.

ential measurement relaxes the control required over systematics from lasers and vibrations. Laser phase noise in this setup arises from the passive laser and can be made smaller than shot noise by using lasers with fractional frequency stability $\sim 10^{-15}$ over $1 \mathrm{~s}$ and phase noise below $-140 \frac{\mathrm{dbc}}{\mathrm{Hz}}$ at a frequency offset $\frac{c}{L} \sim 3 \times 10^{5} \mathrm{~Hz}\left(\frac{1 \mathrm{~km}}{L}\right)$. These performance levels have been demonstrated by lasers locked to high finesse cavities 14]. Laser phase noise in the satellite experiment can also be tackled by using the same passive laser to operate two pairs of atom interferometers along two non-parallel baselines established by a LISA-like three satellite constellation. The differential phase shift along each baseline contains the same phase noise from the passive laser but a different gravitational wave signal. The difference between these phase shifts is free from phase noise and retains the signal. Backgrounds to the detection of gravitational waves at these sensitivities in both terrestrial and space based interferometers were studied in [4] and seem controllable down to shot noise levels. Since these sensitivities are not primarily limited by backgrounds, there are many possibilities for improvement as the atomic techniques advance.

The atom interferometer configurations discussed in this paper will be sensitive to interesting astrophysical and cosmological sources. The mergers of bright gravitational wave sources like white dwarf binaries, intermediate and massive black holes occur between $10^{-3} \mathrm{~Hz}$ 
and $10 \mathrm{~Hz}$ [15] and are inaccessible to LIGO. Moreover, compact solar mass binaries spend long times moving through sub-Hertz frequencies before rapidly coalescing in LIGO's band, increasing the population of these binaries in the sub-Hertz band relative to the number in LIGO's band. Also, the long lifetime of these compact binaries in this band increases the integration time available to see them resulting in a significant enhancement in their detectability.

The $10^{-3} \mathrm{~Hz}-10 \mathrm{~Hz}$ band is also interesting for stochastic gravitational wave searches [15]. The power spectra of gravitational waves from violent events in the Universe at the $\mathrm{TeV}$ scale are typically peaked around $10^{-3} \mathrm{~Hz}-10^{-1} \mathrm{~Hz}$. Furthermore, the energy density $\Omega_{G W}$ in gravitational radiation produced by phenomena such as inflation is flat over several frequency decades. The strain $h$ of the gravitational waves produced by these phenomena is consequently significantly higher at lower frequencies. Since gravitational wave detectors respond to $h$, these sources can be more easily detected at lower frequencies. Gravitational wave detectors in the subHertz band will thus provide a new astrophysical and cosmological probe.

The role of atom interferometers in gravitational wave detection has been previously studied [16, 17, 18, $19,20,21]$ and these authors concluded that atom interferometers would need an unrealistic atom flux to probe interesting regions of the gravitational wave spectrum. Our proposal differs significantly from these efforts owing to the central role played by light pulse atom interferometry in this setup. Previous attempts concentrated on the effect of the gravitational waves on the atom trajectories and did not exploit the critical effects of the gravitational wave on the light pulses. In our setup, the atoms effectively function as test masses to record the effects of the gravitational wave on the propagating laser field. The distance over which the light pulses propagate can be easily increased without altering the size of the individual atom interferometers. This permits a large enhancement to the signal while simultaneously suppressing backgrounds. Although we have attempted to provide a way to control all backgrounds, further work is required to turn these proposals into blueprints for a specific experiment. This approach can allow terrestrial atom interferometers to operate in the $1 \mathrm{~Hz}-10 \mathrm{~Hz}$ band which is inaccessible to instruments like LIGO. Space based atom interferometers can look for gravitational waves in the $10^{-3} \mathrm{~Hz}-1 \mathrm{~Hz}$ band with less stringent demands on spacecraft control.

Acknowledgments.- We would like to thank Mustafa Amin, Asimina Arvanitaki, Roger Blandford, Seth Foreman, David Johnson, Vuk Mandic, Holger Mueller, Stephen Shenker, Robert Wagoner, and Yoshihisa Yamamoto. PWG acknowledges the support of the Mellam Family Graduate Fellowship during part of this work.

[1] M. Kasevich and S. Chu, Phys. Rev. Lett. 67, 181 (1991).

[2] S. Dimopoulos, P. W. Graham, J. M. Hogan and M. A. Kasevich, Phys. Rev. Lett. 98, 111102 (2007) arXiv:gr-qc/0610047.

[3] M. J. Snadden, et al., Phys. Rev. Lett. 81, 971 (1998).

[4] S. Dimopoulos, P. W. Graham, J. Hogan, M. Kasevich and S. Rajendran (to be published).

[5] J. W. Sari and N. F. Ness, Solar Physics Vol. 8, No. 1, 155 (1969).

[6] L. F. Burlaga, Solar Physics Vol. 7, 54 (1968).

[7] J. H. Denschlag, et al., J. Phys. B: At. Mol. Opt. Phys. 35, 3095 (2002).

[8] H. Mueller, S.-w. Chiow, Q. Long, S. Herrmann, S. Chu. to appear.

[9] J.M. McGuirk, et al., Phys. Rev. Lett. 85, 4498 (2000).

[10] M. Weitz, et al., Phys. Rev. Lett. 73, 2563 (1994).

[11] P. Bender et al., LISA Pre Phase A, Second Edition (1998).

[12] M. Kitagawa and M. Ueda, Phys. Rev. A 47, 5138 (1993).

[13] A. K. Tuchman et al., Phys. Rev. A 74, 053821 (2006).

[14] A. D. Ludlow et al., Opt. Lett. 32, 641 (2007) arXiv:physics/0610274 $\mathrm{v} 1$ [physics.optics]].

[15] C. Cutler and K. S. Thorne, arXiv:gr-qc/0204090.

[16] B. Lamine, M. T. Jaekel and S. Reynaud, Eur. Phys. J. D 20, 165 (2002) arXiv:quant-ph/0202086.

[17] R. Y. Chiao and A. D. Speliotopoulos, J. Mod. Opt. 51, 861 (2004).

[18] S. Foffa, A. Gasparini, M. Papucci and R. Sturani, Phys. Rev. D 73, 022001 (2006) arXiv:gr-qc/0407039.

[19] A. Roura, D. R. Brill, B. L. Hu and C. W. Misner, Phys. Rev. D 73, 084018 (2006) arXiv:gr-qc/0409002.

[20] P. Delva, M. C. Angonin and P. T. Proxy, Phys. Lett. A 357, 249 (2006) arXiv:gr-qc/0609075.

[21] G. M. Tino and F. Vetrano, Class. Quant. Grav. 24, 2167 (2007) arXiv:gr-qc/0702118. 